PAPER • OPEN ACCESS

\title{
Binary acoustic trapping in a glass capillary
}

To cite this article: Anna Fornell et al 2021 J. Phys. D: Appl. Phys. 54355401

View the article online for updates and enhancements.

The Electrochemical Society 2021 Virtual Education

Fundamentals of Electrochemistry: Basic Theory and Kinetic Methods Instructed by: Dr. James Noël Sun, Sept 19 \& Mon, Sept 20 at 12h-15h ET

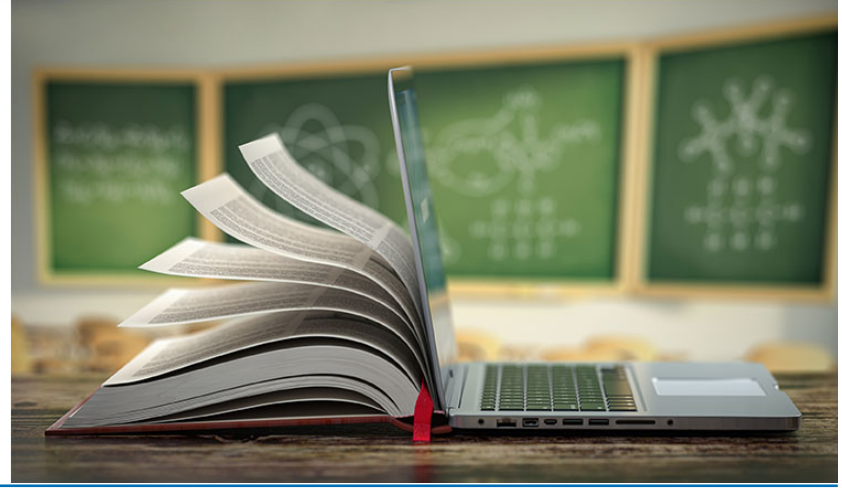




\title{
Binary acoustic trapping in a glass capillary
}

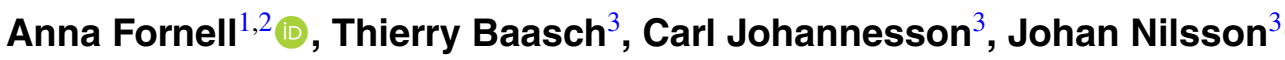 \\ and Maria Tenje ${ }^{1, *}$ (D) \\ ${ }^{1}$ Department of Materials Science and Engineering, Science for Life Laboratory, Uppsala University, \\ 75121 Uppsala, Sweden \\ 2 MAX IV Laboratory, Lund University, 22484 Lund, Sweden \\ ${ }^{3}$ Department of Biomedical Engineering, Lund University, 22100 Lund, Sweden \\ E-mail: maria.tenje@ angstrom.uu.se
}

Received 9 February 2021, revised 21 May 2021

Accepted for publication 4 June 2021

Published 21 June 2021

\begin{abstract}
Acoustic trapping is a useful method for handling biological samples in microfluidic systems. The aim of this work is twofold: first to investigate the physics behind acoustic trapping in a glass capillary and secondly to perform binary acoustic trapping. The latter is achieved by increasing the density of the fluid in the trapping channel. The trapping device consisted of a glass capillary with a rectangular inner cross-section (height $200 \mu \mathrm{m} \times$ width $2000 \mu \mathrm{m}$ ) equipped with a small piezoelectric transducer. The piezoelectric transducer was actuated at $4 \mathrm{MHz}$ to generate a localised half-wavelength acoustic standing-wave-field in the capillary, comprising of a pressure field and a velocity field. Under acoustic actuation, only particles with higher density than the fluid, i.e. having a positive dipole scattering coefficient, were trapped in the flow direction. The numerical and analytical modelling of the system show that the trapping force which retains the particles against the flow depends only on the dipole scattering coefficient in the pressure nodal plane of the acoustic field. The analytical model also reveals that the retention force is proportional to the dipole scattering coefficient, which agrees with our experimental findings. Next, we showed that in a mixture of melamine particles and polystyrene particles in a high-density fluid it is possible to selectively trap melamine particles, since melamine particles have higher density than polystyrene particles.
\end{abstract}

Supplementary material for this article is available online

Keywords: acoustofluidics, separation, trapping, ultrasound

(Some figures may appear in colour only in the online journal)

\section{Introduction}

Microfluidics has emerged as a powerful technology for the analysis of small sample volumes with high sensitivity. In

* Author to whom any correspondence should be addressed.

Original content from this work may be used under the terms of the Creative Commons Attribution 4.0 licence. Any further distribution of this work must maintain attribution to the author(s) and the title of the work, journal citation and DOI recent years, acoustics were integrated into microfluidic systems to enrich, pattern, separate and trap cells and particles [1-4]. Acoustic particle manipulation has the advantages of being label-free, biocompatible [5] and can be operated in noncontact mode. Acoustic particle manipulation can be divided into continuous flow acoustophoresis where particles with different size or density can be sorted [1] and acoustic trapping [2]. In acoustic trapping, the sample is retained against a fluid flow. This has the advantage that the sample can be both enriched and washed in the same device. The applications of acoustic trapping include e.g. enrichment of bacteria 
(a)

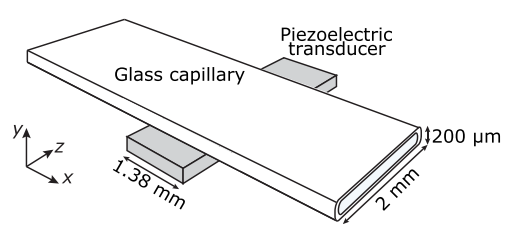

(b)

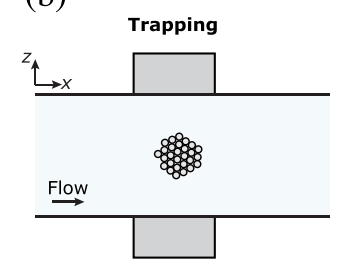

(c)

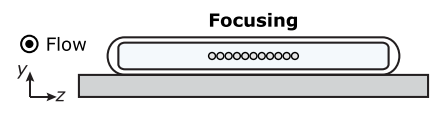

Figure 1. (a) The acoustic trapping device: a glass capillary with a piezoelectric transducer. (b) Acoustic trapping is defined as the retention of the particles against the flow. (c) Acoustic focusing is defined as the movement of particles to the same height in the capillary.

[6] and extracellular vesicles [7], sample preparation for DNA analysis [8], bead-based assays [9], and cell culturing [10, 11].

Acoustic trapping can be realised in different types of microsystems and the principle for acoustic trapping differs slightly between the systems. Glynne-Jones et al [12] presented a system where an array of piezoelectric elements was used to trap particles by actively switching on and off specific piezoelectric elements. This approach allows for localised trapping, but it requires selectively addressable transducers which can be challenging to fabricate. Alternatively, trapping can be realised in a microfluidic resonance cavity by using two external transducers and matching the emitted wavelengths of the ultrasound to the height and width of the cavity, thus generating an acoustic standing-wave-field in the resonance cavity itself [13]. Hammarström et al [14] presented an alternative technical solution where a commercial glass capillary was acoustically actuated by a small piezoelectric transducer. In this set-up, trapping was achieved at a specific position in the capillary since the transducer created a very strong and localised acoustic standing-wave-field due the finite width of the transducer. In this work, the Hammarström approach was followed (figure 1(a)). Due to their cheap manufacturing costs glass capillaries are disposable and thus suitable for clinical applications. Moreover no cleanroom fabrication is needed. Ley and Bruus [15] have studied the acoustic field in glass capillaries using numerical modelling. However, to date there are only limited studies on the physical principles responsible for acoustic trapping and a better understanding of the underlying physics is important to improve the system and find new applications of the technology.

Biological samples are often complex and require separation steps before analysis. In continuous flow acoustophoresis, binary separations of samples have previously been shown. Petersson et al [16] e.g. demonstrated acoustic separation of erythrocytes from lipid particles in blood. Lately, separations based on the acousto-mechanical properties of cells have been further developed using high-density fluids. Augustsson et al [17] demonstrated a technology to measure the acoustomechanical properties of cells using iso-acoustic focusing. Recently, Olofsson et al [18] demonstrated binary acoustic separation of living and dead cells using a high-density fluid. However, there are no reports in the literature on binary acoustic trapping. Here we define 'binary trapping' as selectively trapping of one particle specie in a mixture of two different particle species. Binary trapping allows for faster sample processing and less sample consumption, as the number of processing steps are reduced compared when a separation step is needed prior to trapping.

In an acoustic trap, particles are focused and trapped by the acoustic radiation forces [19]. The acoustic radiation forces can be divided into the primary acoustic radiation force and the secondary acoustic radiation force. The primary acoustic radiation force denotes the force exerted directly from the acoustic field on the particle while the secondary acoustic radiation force is an effect of sound scattering between the particles. Although, the interparticle force is suspected to play an important role in the trapping of very small particles such as extracellular vesicles by a seed particle cluster, it is not responsible for the trapping of the large seed cluster particles themselves. In this work, we limit our investigation to the primary acoustic radiation force, which is in our case responsible for both the focusing and trapping of the particles. In addition to the acoustic radiation forces, viscous dissipation of the acoustic waves leads to the so-called acoustic streaming in the fluid. Streaming makes trapping of submicron particles difficult, and the transition from streaming dominated to primary acoustic radiation dominated movement depends on the system, but it occurs usually for particles with a diameter of 1$2 \mu \mathrm{m}$ [20]. In this work, we used large particles $(10 \mu \mathrm{m})$ meaning that the motion of the particles in the acoustic field is dominated by the primary acoustic radiation force and not the acoustic streaming.

In this study, we investigate the physics behind acoustic particle trapping in a glass capillary by using a highdensity fluid (water with Ficoll). The term 'trapping' is used to describe the retention of particles against the flow ( $x$ axis) and the term 'focusing' is used to describe the movement of particles to the same plane in the height direction ( $y$-axis), figures 1(b) and (c). In the first step, we investigate the contribution of the monopole scattering coefficient $f_{1}$ and dipole scattering coefficient $f_{2}$ to acoustic trapping and acoustic focusing, both experimentally and theoretically. Secondly, we experimentally demonstrate binary acoustic trapping of polystyrene particles and melamine particles by adapting the density of the fluid.

\section{Theory}

Trapping and focusing of particles inside a capillary were investigated using both a numerical and an analytical model. The finite-element model was set up in the COMSOL 


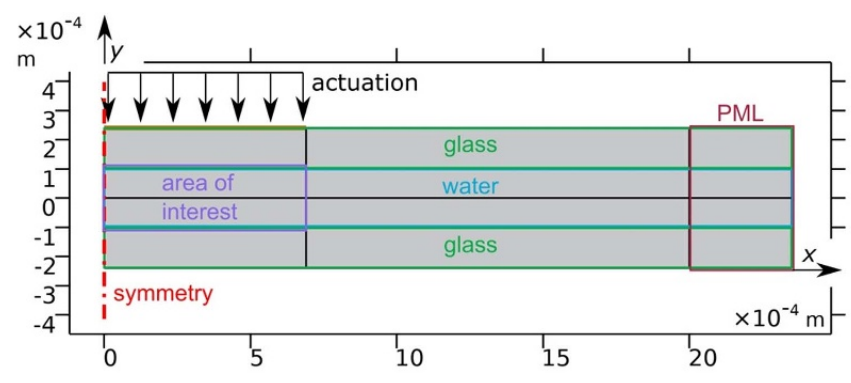

Figure 2. The two dimensional numerical model consists of two glass slides that model the capillary walls, a water channel, and a perfectly matched layer to absorb all outgoing waves and prevent reflections at the (right) end. A symmetry boundary condition is applied to the left boundary. A displacement boundary condition in boundary normal direction models the actuation by the piezoelectric transducer. The area of interest is the water area inside the capillary that extends over the length of the actuation. This is where the strong acoustic fields trap and focus the particles.

Table 1. Material properties for the used materials.

\begin{tabular}{ll}
\hline Parameter & Value \\
\hline Glass & \\
Density $\rho_{\mathrm{gl}}$ & $2230 \mathrm{~kg} \mathrm{~m}^{-3}$ \\
Poisson's ratio $v$ & 0.2 \\
Young's modulus $E$ & $62.75 \mathrm{GPa}$ \\
Water & \\
Density $\rho_{0}$ & $1000 \mathrm{~kg} \mathrm{~m}^{-3}$ \\
Speed of sound $c_{0}$ & $1500 \mathrm{~m} \mathrm{~s}^{-1}$ \\
\hline
\end{tabular}

Multiphysics (version 5.5) software framework (Stockholm, Sweden) and solved in the frequency domain.

\subsection{Setup}

A sketch of the two-dimensional finite-element model of the capillary is shown in figure 2. The height and length dimensions of the capillary are included in the modelling. The width dimension, i.e. the $z$-dimension in figure 1 , of the capillary is neglected in this reduced (two-dimensional) model. We further exploit the symmetry of the setup to reduce the model by half along the length of the capillary. In the experiment, a kerfed piezoelectric transducer that was specifically designed to have a strong $4 \mathrm{MHz}$ thickness mode resonance was used. In the model, to simplify the analysis, the piezoelectric transducer is replaced by a $1 \mathrm{~nm}$ displacement boundary condition in the $y$ direction. The area of interest is defined as the water domain inside the capillary that stretches along the length of the actuation in the $x$ direction as shown in figure 2. This is where the acoustic fields are usually the strongest and the particles will be trapped and focused. The dimensions of the domains in the halved model are (length $\times$ height): $2 \mathrm{~mm} \times 140 \mu \mathrm{m}$ for one glass slide and $2 \mathrm{~mm} \times 200 \mu \mathrm{m}$ for the water channel. The length of the area of interest is $690 \mu \mathrm{m}$ and the length of the perfectly matched layer region is $350 \mu \mathrm{m}$. The material properties of the glass are set to the same values as used by Ley et al [15] and can be found in table 1 .

\subsection{Physical modelling}

The fluid domain is modelled as inviscid, the glass and piezo domains are modelled as linear elastic solids, and the piezoelectric effect is included in the domain of the piezoelectric transducer. The stresses and displacements are continuous in the interface normal direction on the glass-water interface. A symmetry condition is applied to the left boundary of the piezoelectric, glass, and water domains. All other boundaries, i.e. the interfaces between the device and the air are stress-free. The perfectly matched layer is the only damping mechanism in our model.

\subsection{The mesh}

A rectangular mesh is chosen to discretise the geometry. The optimal size of the mesh regarding the trade-off between accuracy and speed was determined by a mesh convergence study (supplementary information available online at stacks.iop.org/JPD/54/355401/mmedia). The quality of the mesh is described by the degree of freedom of the tested mesh. The mesh with the largest degree of freedom is thus the most accurate mesh that we used during our mesh study. For the numerical analysis, we selected the mesh with a degree of freedom of 12817 .

\subsection{Resonance sweep}

In the supplementary information (available online at stacks.iop.org/JPD/54/355401/mmedia), a figure of the logarithm of base 10 of the average acoustic energy inside the area of interest as a function of the excitation frequency is shown. The largest average acoustic energy and thus the resonance frequency is found at $3.878 \mathrm{MHz}$.

The negative gradient of the Gorkov potential $U$ [21] yields the acoustic radiation force on a particle of density $\rho_{\mathrm{p}}$ and compressibility $\kappa_{\mathrm{p}}$ in a fluid of speed of sound $c_{0}$ and compressibility $\kappa_{0}$. The Gorkov potential is given by

$$
\begin{gathered}
U=V_{\mathrm{p}}\left(\frac{f_{1}}{2 \rho_{0} c_{0}^{2}}\left\langle p^{2}\right\rangle-\frac{3}{4} f_{2} \rho_{0}\left\langle v^{2}\right\rangle\right), \\
f_{1}=1-\frac{\kappa_{\mathrm{p}}}{\kappa_{0}}, f_{2}=\frac{2(\bar{\rho}-1)}{2 \bar{\rho}+1}, \bar{\rho}=\frac{\rho_{\mathrm{p}}}{\rho_{0}},
\end{gathered}
$$

where $\langle f(x, y, t)\rangle:=\frac{1}{\tau} \int_{0}^{\tau} f(x, y, t) \mathrm{d} t, \tau=1 / f$ and $V_{\mathrm{p}}$ is the volume of the particle.

The Gorkov potential for $10 \mu \mathrm{m}$ polystyrene particles inside the capillary excited at resonance is shown in the top panel of figure 3 . The Gorkov potential has a local minimum at the centre of the water channel close to the origin of the coordinate system, which indicates a stable equilibrium for the particles under stopped flow. The Gorkov potential is formed by two terms, one proportional to $\left\langle p^{2}\right\rangle$ and another proportional to $\left\langle v^{2}\right\rangle$, both fields are shown in the middle and bottom panels of figure 3 , respectively. Particles with a positive monopole scattering coefficient $f_{1}$ will be focused in the middle of the channel, as shown by the middle panel of 

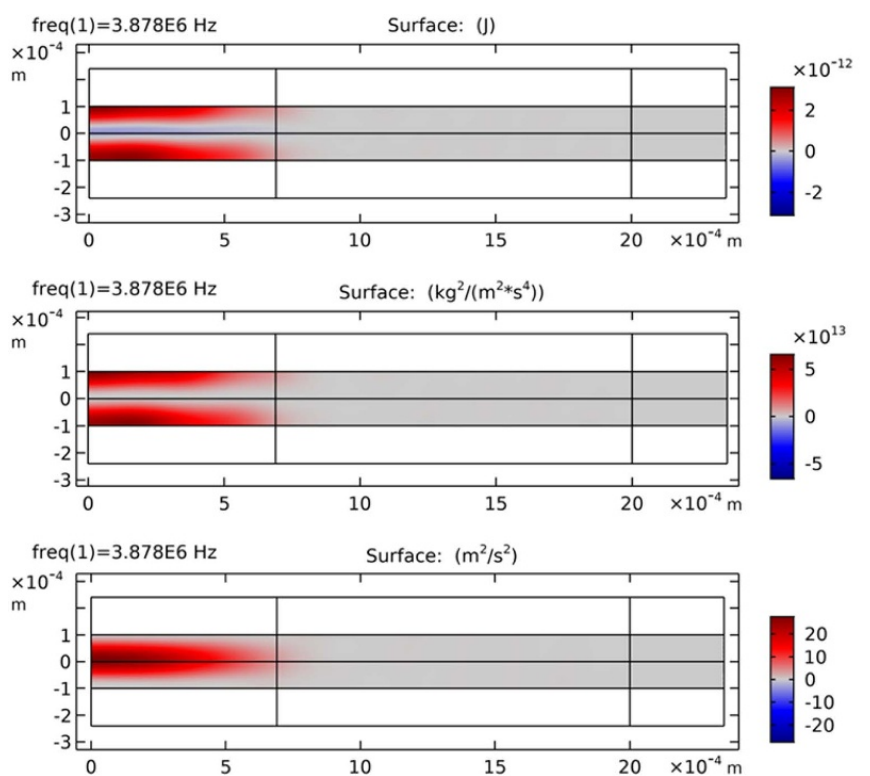

Figure 3. The results are taken from the finite element simulation. (Top) Gorkov potential for $10 \mu \mathrm{m}$ diameter polystyrene particles in water. (Middle) The $\left\langle p^{2}>\right.$ field inside the capillary. (Bottom) The $\left\langle v^{2}\right\rangle$ field inside the capillary. The capillary is excited at the resonance frequency of $3.878 \mathrm{MHz}$.

figure 3. Particles with a positive dipole scattering coefficient $f_{2}$ will be trapped against the flow and collected at the maximum of the $\left\langle v^{2}\right\rangle$ field, as the $\left\langle v^{2}\right\rangle$ field appears with a negative coefficient in the Gorkov potential. The numerical simulation confirms that the dipole scattering coefficient is mostly responsible for the trapping against the flow. To further investigate this phenomenon, we proceeded with a simple mathematical analysis.

\subsection{Analytical model for the acoustic field}

The background pressure field inside the area of interest is assumed to be a two-dimensional standing-wave given by

$$
p(x, y, t)=p_{0} \cos \left(k_{x} x\right) \sin \left(k_{y} y\right) \cos (\omega t)
$$

with the pressure amplitude $p_{0}$, wave numbers $k_{x}$ and $k_{y}$ and angular frequency $\omega$. The pressure field will be a solution of the corresponding wave equation if $k^{2}=k_{x}^{2}+k_{y}^{2}=\left(\frac{\omega}{c}\right)^{2}$, where $c$ is the speed of sound in the surrounding medium. For a medium of density $\rho_{0}$, the corresponding velocity field is given by

$$
\begin{gathered}
\rho_{0} \cdot \frac{\partial}{\partial t} \boldsymbol{v}=-\nabla p, \\
v_{x}(x, y, t)=+\frac{p_{0} k_{x}}{\omega \rho_{0}} \sin \left(k_{x} x\right) \sin \left(k_{y} y\right) \sin (\omega t), \\
v_{y}(x, y, t)=-\frac{p_{0} k_{y}}{\omega \rho_{0}} \cos \left(k_{y} y\right) \cos \left(k_{x} x\right) \sin (\omega t),
\end{gathered}
$$

where $v_{x}$ and $v_{y}$ are the velocity components in the $\mathrm{x}$ and $\mathrm{y}$ direction, respectively.
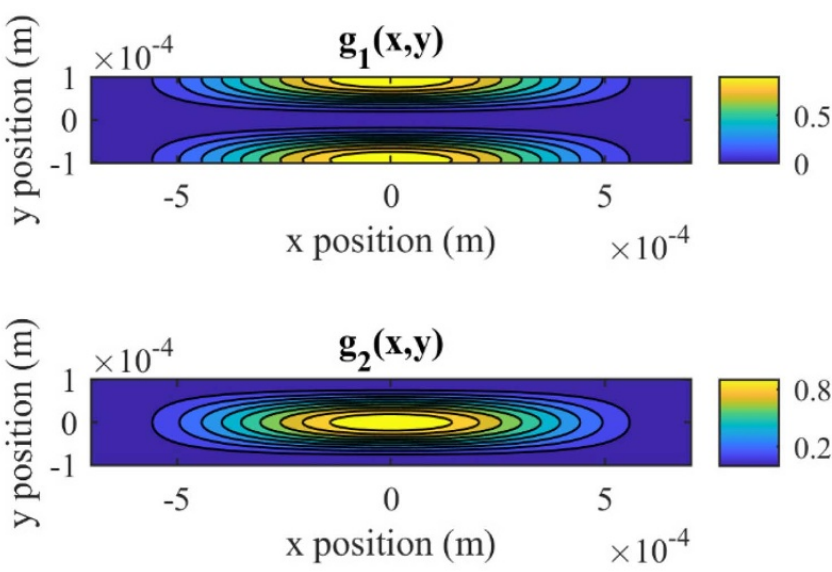

Figure 4. Analytical functions $g_{1}(x, y)$ and $g_{2}(x, y)$ that are used to approximate the acoustic field in the area of interest. Note that we used the same coordinate system as in the modelling of our device in Figures 2 and 3.

We introduce the geometric shape functions $g_{1}(x, y)$ and $g_{2}(x, y)$ via

$$
\begin{gathered}
U=V_{p} \frac{p_{0}^{2}}{4 \rho_{0} c_{0}^{2}}\left(f_{1} g_{1}(x, y)-\frac{3}{2} f_{2} \frac{k_{y}^{2}}{k^{2}} g_{2}(x, y)\right) \\
g_{1}(x, y)=\cos \left(k_{x} x\right)^{2} \sin \left(k_{y} y\right)^{2} \text { and }
\end{gathered}
$$

$g_{2}(x, y)=\cos \left(k_{x} x\right)^{2} \cos \left(k_{y} y\right)^{2}+\frac{k_{x}^{2}}{k_{y}^{2}} \sin \left(k_{x} x\right)^{2} \sin \left(k_{y} y\right)^{2}$.

The wavenumbers $k_{x}$ and $k_{y}$ relate to the respective wavelengths via $\lambda_{x}=\frac{2 \pi}{k_{x}}$ and $\lambda_{y}=\frac{2 \pi}{k_{y}}$ with the additional constraint that $k^{2}=k_{x}^{2}+k_{y}^{2}$. Figure 4 shows the geometrical fields $g_{1}(x, y)$ and $g_{2}(x, y)$ for $k_{x}=2241 \mathrm{rad} \mathrm{m}^{-1}$ and $k_{y}=$ $16605 \mathrm{rad} \mathrm{m}^{-1}$, that correspond to a frequency of $4 \mathrm{MHz}$ and a speed of sound of $c_{0}=1500 \mathrm{~m} \mathrm{~s}^{-1}$. Please note that in our setup $k_{x}^{2} \ll k_{y}^{2}$ and thus the second term of $g_{2}(x, y)$ can be neglected for the subsequent computations. For the approximation to be accurate, the functions $g_{1}(x, y)$ and $g_{2}(x, y)$ should be proportional to the $\left\langle p^{2}\right\rangle$ and $\left\langle v^{2}\right\rangle$ of the simulation in the area of interest, respectively.

The force in $x$ direction is then given by

$$
F_{x}=-\frac{\partial}{\partial x} U
$$

or explicitly

$$
\begin{aligned}
& F_{x}=V_{\mathrm{p}} \frac{f_{1} k_{x} p_{0}^{2}}{2 c_{0}^{2} \rho_{0}} \cos \left(k_{x} x\right) \sin \left(k_{x} x\right) \sin \left(k_{y} y\right)^{2} \\
& -V_{\mathrm{p}} \frac{3 f_{2} p_{0}^{2}}{4 \omega^{2} \rho_{0}} k_{x} k_{y}^{2} \cos \left(k_{x} x\right) \cos \left(k_{y} y\right)^{2} \sin \left(k_{x} x\right)
\end{aligned}
$$




$$
\begin{gathered}
+V_{\mathrm{p}} \frac{3 f_{2} p_{0}^{2}}{4 \omega^{2} \rho_{0}} k_{x}^{3} \cos \left(k_{x} x\right) \sin \left(k_{x} x\right) \sin \left(k_{y} y\right)^{2} \\
\approx V_{\mathrm{p}} \frac{f_{1} k_{x} p_{0}^{2}}{2 c_{0}^{2} \rho_{0}} \cos \left(k_{x} x\right) \sin \left(k_{x} x\right) \sin \left(k_{y} y\right)^{2} \\
-V_{\mathrm{p}} \frac{3 f_{2} p_{0}^{2}}{4 \omega^{2} \rho_{0}} k_{x} k_{y}^{2} \cos \left(k_{x} x\right) \cos \left(k_{y} y\right)^{2} \sin \left(k_{x} x\right) .
\end{gathered}
$$

The retention force $F_{\mathrm{r}}$ is the acoustic radiation force at $y=0$ that acts in $x$ direction, formally

$$
F_{r}=-V_{\mathrm{p}} \frac{3 f_{2} p_{0}^{2}}{4 \omega^{2} \rho_{0}} k_{x} k_{y}^{2} \cos \left(k_{x} x\right) \sin \left(k_{x} x\right) .
$$

Indeed, the retention force only depends on the dipole scattering coefficient $f_{2}$. Thus, the retention force will vanish with vanishing density contrast of the particles with respect to the surrounding medium. Please note, in experiments the stable position of the particles is not exactly in the mid-height of the channel as the acoustic forces need to balance the gravitational forces acting on the particles. As the isolines of the $\left\langle p^{2}\right\rangle$ field are mostly parallel along the channel $x$ direction, this field will not be able to hold the particles against the flow even if they are not positioned exactly at the centre.

\section{Material and methods}

\subsection{Acoustic trapping device}

The acoustic trapping device consisted of a glass capillary (Vitrocom, Mountain Lakes, United States) with a close to rectangular inner cross-section $(200 \mu \mathrm{m} \times 2000 \mu \mathrm{m}$, height $\times$ width). A glass capillary (density $2230 \mathrm{~kg} \mathrm{~m}^{-3}$, speed of sound longitudinal $5592 \mathrm{~m} \mathrm{~s}^{-1}$, speed of sound transverse $3424 \mathrm{~m} \mathrm{~s}^{-1}$ [15]) was selected because it allows for the buildup of strong acoustic resonance. Fluid connectors made of $1 \mathrm{~cm}$ long pieces of Tygon tubing (Saint-Gobain, La Défense, France) were attached to the ends of the capillary. A thin layer of chromium was evaporated on the side of the capillary facing the transducer to enhance the visibility in the trap. The glass capillary was mounted onto a printed circuit board with a small piezoelectric transducer (Pz26, Meggit A/S, Kvistgaard, Denmark). The glass capillary was mounted in a chip holder, and the capillary was positioned in horizontal position (i.e. with the flow direction along the horizontal). The length of the piezoelectric transducer in the flow direction was $1.38 \mathrm{~mm}$. The transducer had a thickness of $0.4 \mathrm{~mm}$, and was kerfed to minimise unwanted resonances [22]. The optimal resonance frequency of the transducer was $4 \mathrm{MHz}$, thus suitable for generation of half-wavelength resonance in the height direction of the capillary. Glycerol was added between the transducer and the capillary to ensure proper acoustic coupling between the layers.

\subsection{Fluids and particles}

Ficoll (Ficoll PM 400, GE Healthcare, Uppsala, Sweden) was added to water to increase the density of the fluid. Water
Table 2. Acoustic properties of fluids and particles used in the experiments.

\begin{tabular}{lccc}
\hline Material & $\begin{array}{c}\text { Density } \rho \\
\left(\mathrm{kg} \mathrm{m}^{-3}\right)\end{array}$ & $\begin{array}{c}\text { Speed of } \\
\text { sound } c \\
\left(\mathrm{~m} \mathrm{~s}^{-1}\right)\end{array}$ & $\begin{array}{c}\text { Compressibility } \\
\kappa\left(\mathrm{Pa}^{-1}\right)\end{array}$ \\
\hline Water & $997^{\mathrm{a}}$ & $1497^{\mathrm{a}}$ & $4.48 \times 10^{-10 \mathrm{~b}}$ \\
14\% Ficoll & $1050^{\mathrm{c}}$ & $1531^{\mathrm{a}}$ & $4.06 \times 10^{-10 \mathrm{~b}}$ \\
$28 \%$ Ficoll & $1094^{\mathrm{a}}$ & $1565^{\mathrm{a}}$ & $3.73 \times 10^{-10 \mathrm{~b}}$ \\
Polystyrene particles & $1050^{\mathrm{c}}$ & $2350^{\mathrm{d}}$ & $2.73 \times 10^{-10 \mathrm{e}}$ \\
Melamine particles & $1500^{\mathrm{e}}$ & $3132^{\mathrm{d}}$ & $1.24 \times 10^{-10 \mathrm{e}}$ \\
& & & \\
\hline
\end{tabular}

a From [23].

${ }^{\mathrm{b}}$ Calculated as $\kappa=1 /\left(c^{2} \rho\right)$.

${ }^{\mathrm{c}}$ Data sheet from the manufacturer (Sigma-Aldrich).

${ }^{\mathrm{d}}$ From [24].

${ }^{\mathrm{e}}$ From [25].

Table 3. Values for the monopole scattering coefficients $\left(f_{1}\right)$ and the dipole scattering coefficients $\left(f_{2}\right)$.

\begin{tabular}{lcr}
\hline Particle and fluid combination & $f_{1}$ & $f_{2}$ \\
\hline Polystyrene particles in water & 0.39 & 0.03 \\
Polystyrene particles in 14\% Ficoll & 0.33 & 0.00 \\
Polystyrene particles in 28\% Ficoll & 0.27 & -0.03 \\
Melamine particles in 14\% Ficoll & 0.69 & 0.22 \\
\hline
\end{tabular}

samples containing $0 \%, 10 \%, 12 \%, 14 \%, 16 \%, 18 \%$ and $28 \%$ Ficoll were prepared. Polystyrene particles (10 $\mu \mathrm{m}$ nonfluorescent particles, Sigma-Aldrich, Stockholm, Sweden or $10 \mu \mathrm{m}$ Fluro-Max green-fluorescent particles, ThermoFisher Scientific, Göteborg, Sweden) and/or melamine particles (10 $\mu \mathrm{m}$, Fluka, Buchs, Switzerland) were suspended in the fluid. The acoustic properties of water, water with $14 \%$ and $28 \%$ Ficoll (hereafter called 14\% Ficoll and 28\% Ficoll), polystyrene particles and melamine particles are shown in table 2. In table 3 the calculated values for the monopole scattering coefficients $\mathrm{f}_{1}$ and dipole scattering coefficients $f_{2}$ are presented.

\subsection{Acoustic operation}

The piezoelectric transducer was actuated by applying a sine signal $\left(10 \mathrm{~V}_{\text {peak-peak }}\right)$ from a function generator (33210A, Keysight, Santa Rosa, United States). The signal over the piezoelectric transducer was monitored with an oscilloscope (TDS1002, Tektronix, Beaverton, United States). The resonance frequency depends on the channel dimensions and the speed of sound of the fluid so that the higher the speed of sound in the fluid, the higher the resonance frequency. The resonance frequency for each fluid composition was determined by identifying the lowest electrical impedance of the attached transducer, a method previously described by Hammarström et al [22]. The resonance frequency of the system was in the region of 4.02-4.13 MHz for the fluids used in these experiments. 


\subsection{Experiments}

Particles and fluid were injected from an Eppendorf tube by a syringe pump (NEMESYS, Cetoni, Korbussen, Germany) operated in withdrawal mode. In all the experiments, fluid without any particles was first injected and the resonance frequency was determined as described in the acoustic operation section.

In the acoustic trapping experiments, the transducer was actuated and fluid with particles was injected at a flow rate of $10 \mu \mathrm{min}^{-1}$, and whether trapping occurred or not was determined by visual inspection by measuring the number of particles at the trapping zone. This was done for polystyrene particles suspended in $0 \%, 10 \%, 12 \%, 14 \%, 16 \%, 18 \%$ and $28 \%$ Ficoll, and for melamine particles suspended in $14 \%$ Ficoll. In these experiments the concentration of particles was $0.05 \mathrm{wt} \%$.

To evaluate if the particles were focused to the same height by the acoustics a high magnification objective $(10 \times)$ with a short depth of field (high numerical aperture) was used. If all particles were sharp in the microscope image, that meant that they were positioned at the same height in the capillary (i.e. the same focal plane). Practically, the evaluation of the acoustic focusing was done by trapping the particles in a cluster, stopping the fluid flow $\left(0 \mu \mathrm{l} \mathrm{min}{ }^{-1}\right)$, then turning off the acoustic actuation and observing the movement of the cluster in the $y$ direction (out of focus). Particles having higher density than the fluid sedimented at the bottom of the capillary (moving downwards, $y$ direction), while particles having lower density than the fluid were floating (moving upwards, $y$ direction). For the cases where the particles were not trapped the positioning of the particles in the height direction was studied under flow $\left(10 \mu 1 \min ^{-1}\right)$.

The binary acoustic trapping experiments were performed by injecting $16 \%$ Ficoll containing a mixture of fluorescent polystyrene particles and melamine particles $\left(10 \mu 1 \mathrm{~min}^{-1}\right)$. The particle fractions in the prepared mixture were $22 \%$ polystyrene particles and $78 \%$ melamine particles. However, the two particle species have different sedimentation rates which unfortunately results in unequal particle losses in the Eppendorf tube and the tubing. Therefore, we chose to quantify the particle ratios of the incoming fluid entering the acoustic trap and these values are given for each replicate in figure 7. Experiments were performed at two different particle concentrations. For the high particle concentration experiment, the concentration of polystyrene particles was $8 \times 10^{-4}$ wt $\%$ and the concentration of melamine particles was $4 \times 10^{-3} \mathrm{wt} \%$. For the low particle concentration experiment, the concentration of polystyrene particles was $1.6 \times 10^{-4} \mathrm{wt} \%$ and the concentration of melamine particles was $8 \times 10^{-4} \mathrm{wt} \%$. The acoustic actuation was applied and the particles were trapped in a cluster. The flow was stopped and images of the cluster were acquired in bright field and fluorescent mode.

\subsection{Microscopy and analysis}

All experiments were performed in an optical microscope (BX51W1, Olympus, Tokyo, Japan) equipped with a camera
(XM10, Olympus, Tokyo, Japan). To measure the binary trapping performance the ratio between fluorescent polystyrene particles and melamine particles in the fluid was first determined by image analysis. This was done by acquiring movies in bright field and fluorescent mode before each experiment, and then manually counting the particles in the fluid that passed the trapping area (no acoustics applied). Only the polystyrene particles were visible in fluorescent mode while both particle species were visible in bright field mode, hence the ratio could be determined. The ratio of the two particle species trapped in the cluster was determined by manually counting the two particle species in one bright field and one fluorescent image of the same stationary cluster. The results were quantified by calculating the reduction in concentration of polystyrene particles in the trap compared with the incoming fluid:

$$
\text { Reduction }=\frac{\mathrm{PS}_{\text {fluid }}-\mathrm{PS}_{\text {trap }}}{\mathrm{PS}_{\text {fluid }}}
$$

where $\mathrm{PS}_{\text {fluid }}$ is the ratio of polystyrene particles compared with the total number of particles in the fluid, and $\mathrm{PS}_{\text {trap }}$ is the ratio of polystyrene particles compared with the total number of particles in the trap.

\section{Results and discussion}

In the first set of experiments, acoustic trapping of polystyrene particles in different concentrations of Ficoll was investigated. In de-ionised water, the polystyrene particles were trapped by the acoustics (figure 5(a)). However, polystyrene particles in $14 \%$ Ficoll (neutrally buoyant) were not trapped by the acoustics (figure 5(b)). Neither were polystyrene particles in $28 \%$ Ficoll trapped by the acoustics (figure 5(c)). Movies of trapping of polystyrene particles in water and in $28 \%$ Ficoll are available in the supplementary information (available online at stacks.iop.org/JPD/54/355401/mmedia). Experiments with other concentrations of Ficoll show similar results. At $10 \%$ Ficoll, a cluster was trapped whereas at higher concentration of Ficoll, no trapping occurred (supplementary information available online at stacks.iop.org/JPD/54/355401/mmedia). A control experiment with melamine particles in $14 \%$ Ficoll was performed, and as seen in figure 5(d) the melamine particles were trapped by the acoustics. As expected, the resonance frequency increased with the concentration of Ficoll and this was compensated for in the set up via the frequency adjustment procedure discussed above. In figure 5(c) it can be seen that the particles align into several bands along the flow direction. This is something that is sometimes seen in this type of trapping devices, and it is probably related to lateral resonance modes. The effect is similar to continuous flow systems where one transducer can be used to set up a standing-wave-field in both height and width direction in the channel to focus the particles [26]. However, the formation of banding in the lateral direction in the acoustic trap has not been further examined in the current work.

In the second set of experiments, focusing of particles in the height direction was investigated. Under acoustic actuation polystyrene particles in water and melamine particles in $14 \%$ 
(a) Polystyrene particles in water
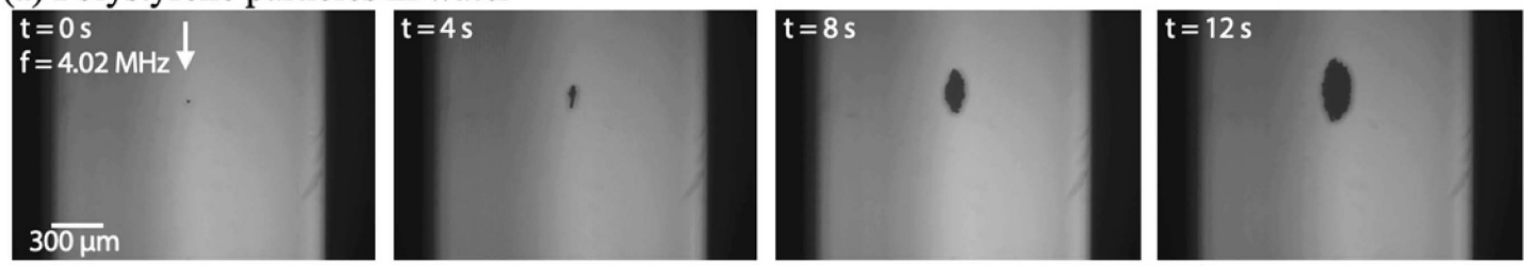

(b) Polystyrene particles in 14\% Ficoll
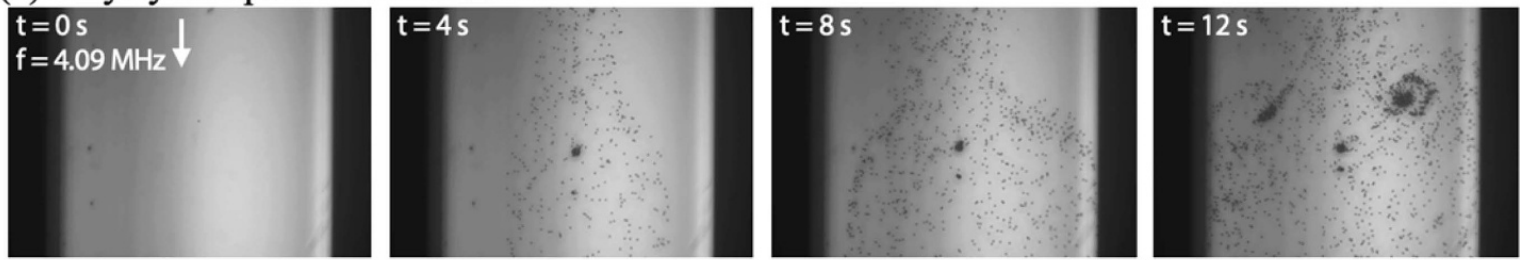

(c) Polystyrene particles in 28\% Ficoll
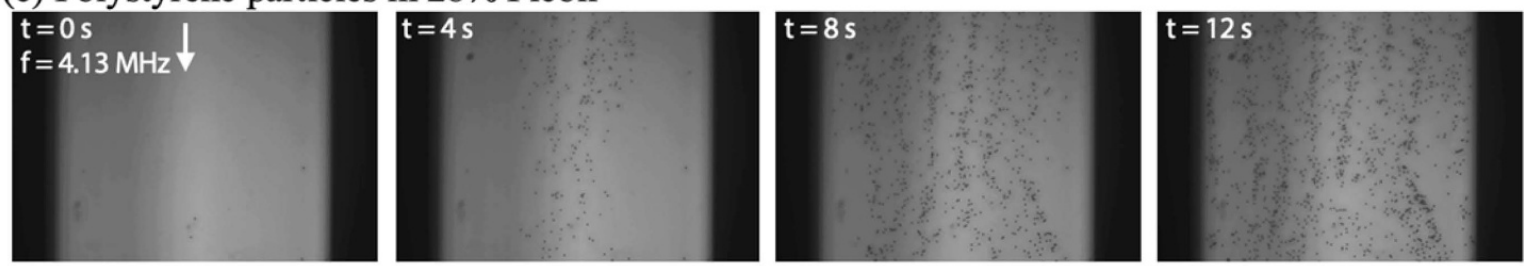

(d) Melamine particles in 14\% Ficoll
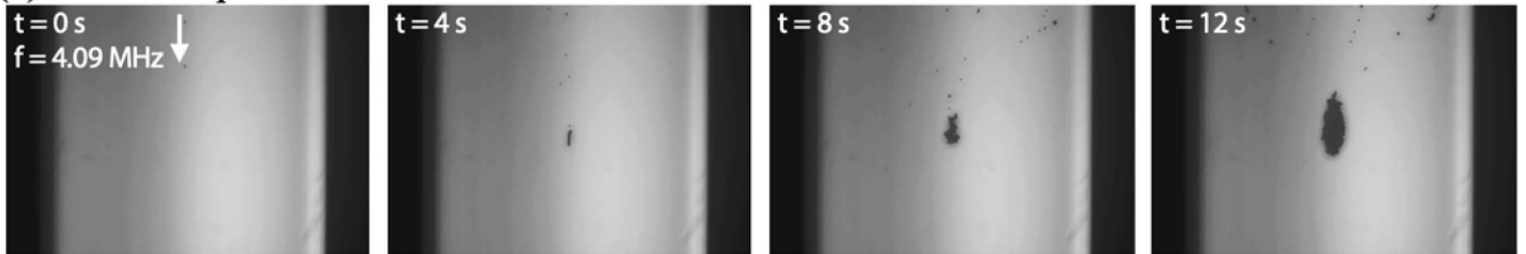

Figure 5. Top-view images of acoustic trapping. In all images acoustics is applied. The flow direction is from the top to the bottom in all images as indicated by the white arrows. The length of the transducer in the flow direction is $1.38 \mathrm{~mm}$, and hence it covers most of the area shown in the pictures. The scale is the same for all images.

(a) Polystyrene particles in water
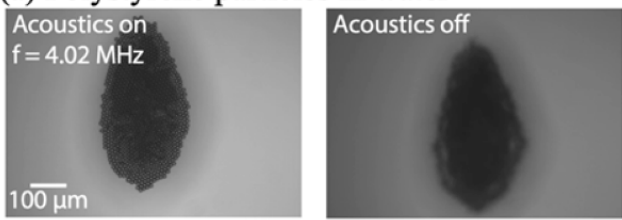

(c) Polystyrene particles in $28 \%$ Ficoll
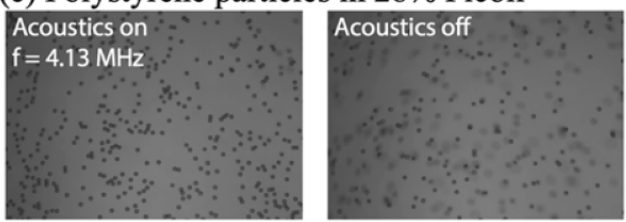

(b) Polystyrene particles in $14 \%$ Ficoll
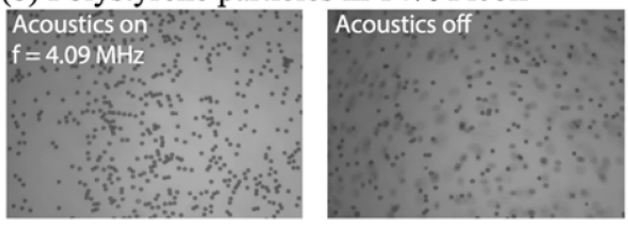

(d) Melamine particles in $14 \%$ Ficoll
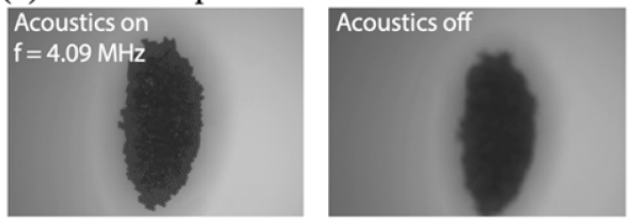

Figure 6. Top-view images of acoustic focusing in the height direction. The scale is the same for all images.

Ficoll formed a cluster, and when the acoustics was turned off the clusters sedimented and moved out of focus (figures 6(a) and (d) right). Polystyrene particles in 14\% Ficoll and $28 \%$ Ficoll were not trapped by the acoustics, whereas the acoustics caused all particles to be positioned in the same focal plane (figures 6(b) and (c) left). Without the acoustics applied, the polystyrene particles in 14\% Ficoll and 28\% Ficoll were positioned randomly in the height direction and only a few particles were in the focal plane (figures 6(b) and (c) right). Experiments with polystyrene particles suspended in other 
(a)

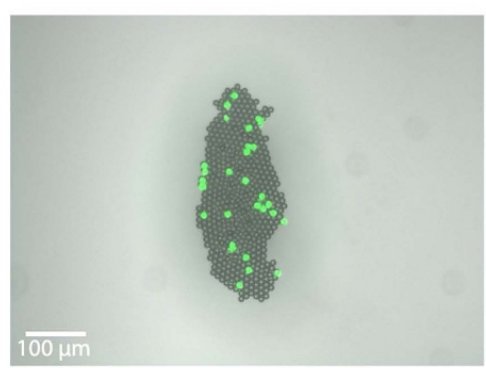

(b)

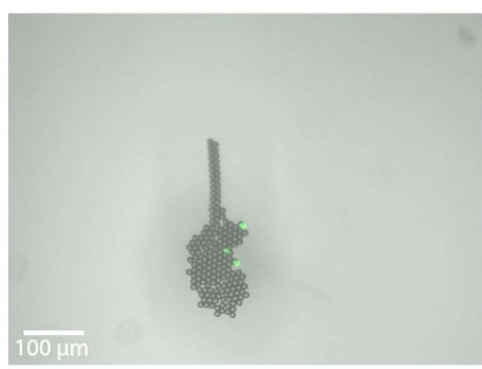

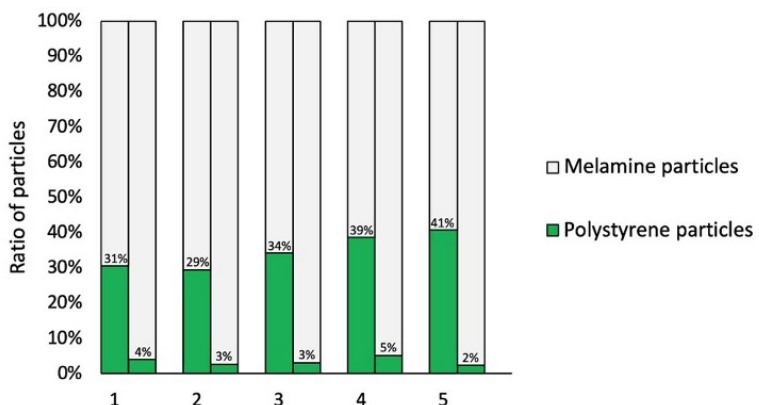

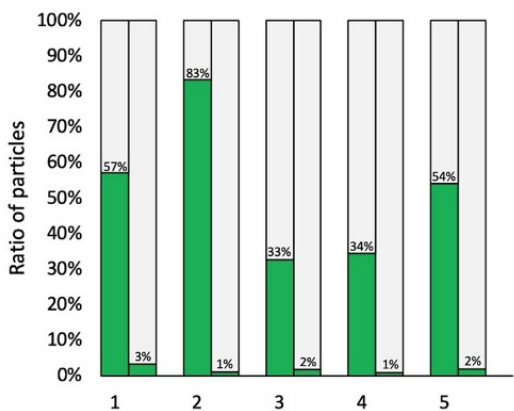

口Melamine particles

๑Polystyrene particles

Figure 7. (a) Acoustic binary trapping at high total particle concentration in the fluid $(n=5)$. (b) Acoustic binary trapping at low total particle concentration in the fluid $(n=5)$. In the photos the green-fluorescent particles are the polystyrene particles and the non-fluorescent particles are the melamine particles. In the graph for each replicate the first bar gives the ratio of the two particle species in the incoming fluid and the second bar shows the ratio of the two particle species in the trapped cluster.

concentrations of Ficoll resulted in the particles being focused by the acoustics (supplementary information available online at stacks.iop.org/JPD/54/355401/mmedia)).

The first set of experiments shows that for acoustic trapping to occur the particles need to have a higher density than the fluid (dipole scattering coefficient $f_{2}>0$ ). This agrees with both the numerical and analytical models as discussed above. In the analytical model (equation (16) it can be seen that the retention force which is responsible for trapping the particles against the flow only depends on the dipole scattering coefficient $f_{2}$ and not on the monopole scattering coefficient $f_{1}$. As the density difference between the particles and the fluid vanishes, the retention force will also vanish, and this effect was observed in the experiments (polystyrene particles in $14 \%$ Ficoll). For particles with lower density than the fluid (dipole scattering coefficient $f_{2}<0$ ) the analytical model shows that the retention force will have the opposite sign and hence no trapping will occur. This was also seen in the experiments (polystyrene particles in 28\% Ficoll). In the second set of experiments it was shown that even if the particles were not trapped, particles with higher, the same or lower density than the fluid could all be focused in the height direction by the acoustics. The numerical simulations (figure 3 middle panel) confirm that particles with the monopole scattering coefficient $f_{1}>0$ will be focused to the mid-height of the capillary.

Based on these findings binary acoustic trapping was investigated. A mixture of polystyrene particles and melamine particles in $16 \%$ Ficoll was introduced into the capillary $\left(10 \mu \mathrm{lmin}^{-1}\right) .16 \%$ Ficoll was used instead of $14 \%$ Ficoll to make the system less sensitive to uncertainties arsing when preparing the Ficoll solution or errors occurring when measuring the acoustic properties of the particles or fluids. Under acoustic actuation (4.08 MHz) some particles were moved towards the trapped cluster whereas other particles were repelled from the area (a movie is available in the supplementary information available online at stacks.iop.org/JPD/ 54/355401/mmedia). The trapped cluster contained mainly melamine particles (figure 7). The ratio of polystyrene particles and melamine particles in the fluid and the cluster for five replicates are presented for two different particle concentrations in figure 7. All replicates were done in exactly the same manner: the same solution of particles and vortexed immediately before the experiment, although there is a significant difference in the concentration of particles entering the trapping area (most noticeable in replicate 2 at low particle concentration). This variation can be explained by the different sedimentation rate of the particle species. The reduction of trapped polystyrene particles was $89.9 \pm 3.1 \%$ (s.d.) and $96.3 \pm 1.8 \%$ (s.d.) for the high and low concentration of particles, respectively. Thus, the results indicate that at lower total particle concentration the reduction in the ratio of trapped polystyrene particles is improved.

Binary trapping is a very useful tool in various applications as it allows for separation, enrichment and washing at the same time in one device. Future experiments involve determining how large the density difference between the two particle species needs to be for the particles to be selectively trapped. 


\section{Conclusion}

In this work, acoustic trapping of particles in high-density fluids was investigated. In the experiments it was found that only particles having higher density than the fluid (positive dipole scattering coefficient $f_{2}>0$ ) were trapped in the flow direction. This result was supported by numerical and analytical modelling of the system, showing that the trapping force depends only on the dipole scattering coefficient $f_{2}$. As the density difference between the particles and the fluid vanishes, the retention force will also vanish. However, particles having higher, the same and lower density than the fluid were focused in the height direction. Based on the principle that the particles need to have positive dipole scattering coefficient $f_{2}$ for trapping to occur, binary trapping was demonstrated. A mixture of polystyrene particles and melamine particles was suspended in a high-density fluid (16\% Ficoll), and in the acoustic trap mainly the higher density melamine particles were trapped.

\section{Data availability statement}

All data that support the findings of this study are included within the article (and any supplementary files).

\section{Acknowledgments}

This project has received funding from the European Research Council (ERC) under the European Union's Horizon 2020 research and innovation programme (Grant Agreement No. 757444) and the Knut and Alice Wallenberg Foundation (WAF 2016.0112). The authors would like to thank Per Augustsson and Mikael Evander (Lund University) for valuable discussions.

\section{ORCID iDs}

Anna Fornell (D) https://orcid.org/0000-0001-7980-376X Johan Nilsson (i) https://orcid.org/0000-0003-1282-9580 Maria Tenje (D) https://orcid.org/0000-0002-1264-1337

\section{References}

[1] Lenshof A, Magnusson C and Laurell T 2012 Lab Chip 12 1210-23

[2] Evander M and Nilsson J 2012 Lab Chip 12 4667-76

[3] Kang P et al 2020 Lab Chip 20 987-94

[4] Zhao S et al 2020 Lab Chip 20 1298-308

[5] Wiklund M 2012 Lab Chip 12 2018-28

[6] Hammarström B, Laurell T and Nilsson J 2012 Lab Chip 12 4296-304

[7] Ku A, Lim H C, Evander M, Lilja H, Laurell T, Scheding S and Ceder Y 2018 Anal. Chem. 90 8011-9

[8] Norris J V, Evander M, Horsman-Hall K M, Nilsson J, Laurell T and Landers J P 2009 Anal. Chem. 81 6089-95

[9] Tenje M, Xia H, Evander M, Hammarström B, Tojo A, Belák S, Laurell T and LeBlanc N 2015 Anal. Chim. Acta 853 682-8

[10] Christakou A E, Ohlin M, Önfelt B and Wiklund M 2015 Lab Chip 15 3222-31

[11] Tait A et al 2019 Sci. Rep. 9 1-11

[12] Glynne-Jones P, Démoré C E M, Ye C, Qiu Y, Cochran S and Hill M 2012 IEEE Trans. Ultrason. Ferroelectr. Freq. Control 59 1258-66

[13] Manneberg O, Vanherberghen B, Svennebring J, Hertz H M, Önfelt B and Wiklund M 2008 Appl. Phys. Lett. 93063901

[14] Hammarström B, Evander M, Barbeau H, Bruzelius M, Larsson J, Laurell T and Nilsson J 2010 Lab Chip 10 2251-7

[15] Ley M W H and Bruus H 2017 Phys. Rev. Appl. 8024020

[16] Petersson F, Nilsson A, Holm C, Jonsson H and Laurell T 2005 Lab Chip 5 20-22

[17] Augustsson P, Karlsen J T, Su H-W, Bruus H and Voldman J 2016 Nat. Commun. 711556

[18] Olofsson K, Hammarström B and Wiklund M 2020 Lab Chip 20 1981-90

[19] Bruus H 2012 Lab Chip 12 1014-21

[20] Barnkob R, Augustsson P, Laurell T and Bruus H 2012 Phys. Rev. E 86056307

[21] Gor'kov L P 1962 Sov. Phys. Dokl. 6 773-5

[22] Hammarström B, Evander M, Wahlström J and Nilsson J 2014 Lab Chip 14 1005-13

[23] Qiu W, Karlsen J T, Bruus H and Augustsson P 2019 Phys. Rev. Appl. 11024018

[24] Settnes M and Bruus H 2012 Phys. Rev. E 85016327

[25] Cushing K W, Garofalo F, Magnusson C, Ekblad L, Bruus H and Laurell T 2017 Anal. Chem. 89 8917-23

[26] Augustsson P, Magnusson C, Nordin M, Lilja H and Laurell T 2012 Anal. Chem. 84 7954-62 